\title{
Spacelike intersection curve of three spacelike hypersurfaces in $E_{1}^{4}$
}

\begin{abstract}
In this paper, we compute the Frenet vectors and the curvatures of the spacelike intersection curve of three spacelike hypersurfaces given by their parametric equations in four-dimensional Minkowski space $E_{1}^{4}$.
\end{abstract}

1. Introduction. The surface-surface intersection(SSI) is one of the basic problems in computational geometry. The main purpose here is to determine the intersection curve between the surfaces and to get information about the geometrical properties of the curve. Since the surfaces are mostly given by their parametric or implicit equations, three cases are valid for the SSI problems: parametric-parametric, implicit-implicit and parametricimplicit.

There are two types of SSI problems: transversal or tangential. The intersection at the intersecting points is called transversal if the normal vectors of the surfaces are linearly independent, and is called tangential if the normal vectors of the surfaces are linearly dependent. The tangent vector of the intersection curve can be obtained easily by the vector product of the normal vectors of the surfaces in transversal intersection problems. Therefore, so many studies have recently been done about this type of problems. Hartmann [6], provides formulas for computing the curvatures of the intersection curves for all types of intersection problems in three-dimensional Euclidean space. Willmore [11], and using the implicit function theorem

2010 Mathematics Subject Classification. 53A35, 53A04, 53A05.

Key words and phrases. Intersection curve, hypersurface. 
Alessio [1], give the methods to compute the unit tangent, the unit principal normal, the unit binormal vectors, and then the curvature and the torsion of the transversal intersection curve of two implicit surfaces. Goldman [5], provides formulas for computing the curvature and the torsion of intersection curve of two implicit surfaces, using the classical curvature formulas in Differential Geometry. Ye and Maekawa [12], present algorithms to compute the Frenet vectors and curvatures of the intersection curve for all three types of transversal and tangential intersections. Alessio and Guadalupe [2], give formulas for computing the local properties of a transversal intersection curve of two spacelike surfaces in the Lorentz-Minkowski 3-space. Using the implicit function theorem, Alessio [3], presents algorithms for computing the differential geometry properties of intersection curves of three implicit surfaces in $R^{4}$. Düldül [4], gives methods for computing the Frenet apparatus of the transversal intersection curve of three parametric hypersurfaces in four-dimensional Euclidean space.

In this paper, we find the tangent, the principal normal, the first and second binormal vectors and the first, second and third curvatures of the spacelike intersection curve of three parametric spacelike hypersurfaces which are intersecting transversally in $E_{1}^{4}$.

2. Preliminaries. The Minkowski space $E_{1}^{4}$ is the real vector space $R^{4}$ provided with the (standard flat) metric given by

$$
g=-d x_{1}^{2}+d x_{2}^{2}+d x_{3}^{2}+d x_{4}^{2},
$$

where $\left(x_{1}, x_{2}, x_{3}, x_{4}\right)$ is a rectangular coordinate system of $E_{1}^{4}$. A vector $\mathbf{u} \in E_{1}^{4}$ is called a spacelike, a timelike, and a null (lightlike) vector if $g(\mathbf{u}, \mathbf{u})>0$ or $\mathbf{u}=0, g(\mathbf{u}, \mathbf{u})<0$, and $g(\mathbf{u}, \mathbf{u})=0$ for $\mathbf{u} \neq 0$, respectively, [8]. The norm of a vector $\mathbf{u}$ is defined by $\|\mathbf{u}\|=\sqrt{|g(\mathbf{u}, \mathbf{u})|}$. Two vectors $\mathbf{u}$ and $\mathbf{v}$ are said to be orthogonal if $g(\mathbf{u}, \mathbf{v})=0$. A vector $\mathbf{u}$ satisfying $g(\mathbf{u}, \mathbf{u})= \pm 1$ is called a unit vector. For an arbitrary curve $\alpha=\alpha(s)$ in $E_{1}^{4}$, if all of its velocity vectors $\alpha^{\prime}(s)$ are spacelike, timelike or null vectors, then the curve is called a spacelike, a timelike or a null curve, respectively, [8].

A hypersurface in $E_{1}^{4}$ is called a timelike (spacelike) hypersurface if the induced metric on the hypersurface is a Lorentz (positive definite Riemannian) metric. The normal vector on the timelike (spacelike) hypersurface is a spacelike (timelike) vector.

The ternary product of the vectors $\mathbf{u}=\sum_{i=1}^{4} u_{i} \mathbf{e}_{i}, \mathbf{v}=\sum_{i=1}^{4} v_{i} \mathbf{e}_{i}$, and $\mathbf{w}=\sum_{i=1}^{4} w_{i} \mathbf{e}_{i}$ is defined by

$$
\mathbf{u} \otimes \mathbf{v} \otimes \mathbf{w}=-\left|\begin{array}{cccc}
-\mathbf{e}_{1} & \mathbf{e}_{2} & \mathbf{e}_{3} & \mathbf{e}_{4} \\
u_{1} & u_{2} & u_{3} & u_{4} \\
v_{1} & v_{2} & v_{3} & v_{4} \\
w_{1} & w_{2} & w_{3} & w_{4}
\end{array}\right|,
$$


where $\left\{\mathbf{e}_{1}, \mathbf{e}_{2}, \mathbf{e}_{3}, \mathbf{e}_{4}\right\}$ is the standard basis of four-dimensional Minkowski space $E_{1}^{4}$.

For the vectors $\mathbf{e}_{1}, \mathbf{e}_{2}, \mathbf{e}_{3}, \mathbf{e}_{4}$ the following equations are satisfied:

$\mathbf{e}_{1} \otimes \mathbf{e}_{2} \otimes \mathbf{e}_{3}=\mathbf{e}_{4}, \mathbf{e}_{2} \otimes \mathbf{e}_{3} \otimes \mathbf{e}_{4}=\mathbf{e}_{1}, \mathbf{e}_{3} \otimes \mathbf{e}_{4} \otimes \mathbf{e}_{1}=\mathbf{e}_{2}, \mathbf{e}_{4} \otimes \mathbf{e}_{1} \otimes \mathbf{e}_{2}=-\mathbf{e}_{3}$, [13].

From the definition of ternary product, we get $g(\mathbf{u}, \mathbf{u} \otimes \mathbf{v} \otimes \mathbf{w})=g(\mathbf{v}, \mathbf{u} \otimes$ $\mathbf{v} \otimes \mathbf{w})=g(\mathbf{w}, \mathbf{u} \otimes \mathbf{v} \otimes \mathbf{w})=0$; that is, the vector $\mathbf{u} \otimes \mathbf{v} \otimes \mathbf{w}$ is orthogonal to $\mathbf{u}, \mathbf{v}$ and $\mathbf{w}$. The triple vector product of vectors $\mathbf{u}, \mathbf{v}$ and $\mathbf{w}$ in $E_{1}^{3}$ is defined by

$$
(\mathbf{u} \times \mathbf{v}) \times \mathbf{w}=\left|\begin{array}{cc}
\mathbf{u} & \mathbf{v} \\
g(\mathbf{u}, \mathbf{w}) & g(\mathbf{v}, \mathbf{w})
\end{array}\right|=g(\mathbf{v}, \mathbf{w}) \mathbf{u}-g(\mathbf{u}, \mathbf{w}) \mathbf{v},
$$

a linear combination of vectors $\mathbf{u}$ and $\mathbf{v},[7,10]$. In an analogous manner in $E_{1}^{4}$ we can express the quintuple vector product of vectors $\mathbf{u}, \mathbf{v}, \mathbf{w}, \mathbf{x}$, and $\mathbf{y}$ as

$$
(\mathbf{u} \otimes \mathbf{v} \otimes \mathbf{w}) \otimes \mathbf{x} \otimes \mathbf{y}=\left|\begin{array}{ccc}
\mathbf{u} & \mathbf{v} & \mathbf{w} \\
g(\mathbf{u}, \mathbf{x}) & g(\mathbf{v}, \mathbf{x}) & g(\mathbf{w}, \mathbf{x}) \\
g(\mathbf{u}, \mathbf{y}) & g(\mathbf{v}, \mathbf{y}) & g(\mathbf{w}, \mathbf{y})
\end{array}\right|,
$$

a linear combination of vectors $\mathbf{u}, \mathbf{v}$ and $\mathbf{w}$ (see [10] for the Euclidean case). We may also write

$$
g(\mathbf{u} \otimes \mathbf{v} \otimes \mathbf{w}, \mathbf{x} \otimes \mathbf{y} \otimes \mathbf{z})=-\left|\begin{array}{ccc}
g(\mathbf{u}, \mathbf{x}) & g(\mathbf{v}, \mathbf{x}) & g(\mathbf{w}, \mathbf{x}) \\
g(\mathbf{u}, \mathbf{y}) & g(\mathbf{v}, \mathbf{y}) & g(\mathbf{w}, \mathbf{y}) \\
g(\mathbf{u}, \mathbf{z}) & g(\mathbf{v}, \mathbf{z}) & g(\mathbf{w}, \mathbf{z})
\end{array}\right| .
$$

Let $\left\{\mathbf{t}(s), \mathbf{n}(s), \mathbf{b}_{1}(s), \mathbf{b}_{2}(s)\right\}$ be the moving Frenet frame along the curve $\alpha(s)$ in the Minkowski 4-space $E_{1}^{4}$. Then $\mathbf{t}(s), \mathbf{n}(s), \mathbf{b}_{1}(s), \mathbf{b}_{2}(s)$ denote the tangent, the principal normal, the first binormal, and the second binormal vector fields, respectively.

Let $\alpha(s)$ be a spacelike curve with arc length parameter $s$ in $E_{1}^{4}$. Then $\mathbf{t}(s)=\alpha^{\prime}(s)$ is a spacelike unit vector, i.e., $\left\|\alpha^{\prime}(s)\right\|=1$. Therefore $g\left(\alpha^{\prime}(s), \alpha^{\prime}(s)\right)=1$ and $g\left(\alpha^{\prime}(s), \alpha^{\prime \prime}(s)\right)=0$. Depending on the vector $\alpha^{\prime \prime}(s)$ we investigate the following cases [9].

Case 1: $\mathbf{n}$ is spacelike:

Case 1.1: The second binormal vector $\mathbf{b}_{2}$ is the unique timelike vector in the Frenet frame $\left\{\mathbf{t}, \mathbf{n}, \mathbf{b}_{1}, \mathbf{b}_{2}\right\}$. Then the Frenet formulas are

$$
\left\{\begin{array}{l}
\mathbf{t}^{\prime}=k_{1} \mathbf{n} \\
\mathbf{n}^{\prime}=-k_{1} \mathbf{t}+k_{2} \mathbf{b}_{\mathbf{1}} \\
\mathbf{b}_{1}^{\prime}=-k_{2} \mathbf{n}+k_{3} \mathbf{b}_{\mathbf{2}} \\
\mathbf{b}_{2}^{\prime}=k_{3} \mathbf{b}_{\mathbf{1}},
\end{array}\right.
$$

where $k_{i}$ 's $(i=1,2,3)$ are the $i t h$ curvature functions of the curve $\alpha$.

Case 1.2: The first binormal vector $\mathbf{b}_{1}$ is the unique timelike vector in the tetrad $\left\{\mathbf{t}, \mathbf{n}, \mathbf{b}_{1}, \mathbf{b}_{2}\right\}$. In this case, the Frenet formulas are given by 
Case 2: $\mathbf{n}$ is timelike:

$$
\left\{\begin{array}{l}
\mathbf{t}^{\prime}=k_{1} \mathbf{n} \\
\mathbf{n}^{\prime}=-k_{1} \mathbf{t}+k_{2} \mathbf{b}_{\mathbf{1}} \\
\mathbf{b}_{1}^{\prime}=k_{2} \mathbf{n}+k_{3} \mathbf{b}_{\mathbf{2}} \\
\mathbf{b}_{2}^{\prime}=-k_{3} \mathbf{b}_{\mathbf{1}}
\end{array}\right.
$$

The Frenet formulas have the form

$$
\left\{\begin{array}{l}
\mathbf{t}^{\prime}=k_{1} \mathbf{n} \\
\mathbf{n}^{\prime}=k_{1} \mathbf{t}+k_{2} \mathbf{b}_{\mathbf{1}} \\
\mathbf{b}_{1}^{\prime}=k_{2} \mathbf{n}+k_{3} \mathbf{b}_{\mathbf{2}} \\
\mathbf{b}_{2}^{\prime}=-k_{3} \mathbf{b}_{\mathbf{1}} .
\end{array}\right.
$$

From elementary differential geometry, we know that $\alpha^{\prime}(s)=\mathbf{t}(s)$. Also, the second derivative $\alpha^{\prime \prime}(s)$ is equal to $k_{1} \mathbf{n}$ in all above cases. The third derivative $\alpha^{\prime \prime \prime}(s)$ can be obtained as $\alpha^{\prime \prime \prime}=k_{1}^{\prime} \mathbf{n}+k_{1} \mathbf{n}^{\prime}$ by differentiating the equation $\alpha^{\prime \prime}=k_{1} \mathbf{n}$. If we replace $\mathbf{n}^{\prime}$ using the Frenet formulas, we get

Case 1:

$$
\alpha^{\prime \prime \prime}=-k_{1}^{2} \mathbf{t}+k_{1}^{\prime} \mathbf{n}+k_{1} k_{2} \mathbf{b}_{1}
$$

Case 2:

$$
\alpha^{\prime \prime \prime}=k_{1}^{2} \mathbf{t}+k_{1}^{\prime} \mathbf{n}+k_{1} k_{2} \mathbf{b}_{1} .
$$

Then the second curvature $k_{2}$ of the curve $\alpha$ can be obtained from Eq. (2.1) and Eq. (2.2) as

Case 1.1 and Case 2: $k_{2}=\frac{g\left(\alpha^{\prime \prime \prime}, \mathbf{b}_{1}\right)}{k_{1}}$.

Case 1.2: $k_{2}=-\frac{g\left(\alpha^{\prime \prime \prime}, \mathbf{b}_{1}\right)}{k_{1}}$.

Now let us find the fourth derivative $\alpha^{(4)}(s)$ similar to the third derivative $\alpha^{\prime \prime \prime}(s)$ :

$$
\text { Case 1.1: } \begin{aligned}
\alpha^{(4)} & =-3 k_{1} k_{1}^{\prime} \mathbf{t}+\left(-k_{1}^{3}+k_{1}^{\prime \prime}-k_{1} k_{2}^{2}\right) \mathbf{n}+\left(2 k_{1}^{\prime} k_{2}+k_{1} k_{2}^{\prime}\right) \mathbf{b}_{1} \\
& +k_{1} k_{2} k_{3} \mathbf{b}_{2}
\end{aligned}
$$

Case 1.2: $\alpha^{(4)}=-3 k_{1} k_{1}^{\prime} \mathbf{t}+\left(-k_{1}^{3}+k_{1}^{\prime \prime}+k_{1} k_{2}^{2}\right) \mathbf{n}+\left(2 k_{1}^{\prime} k_{2}+k_{1} k_{2}^{\prime}\right) \mathbf{b}_{1}$

$$
+k_{1} k_{2} k_{3} \mathbf{b}_{2}
$$

Case 2: $\alpha^{(4)}=3 k_{1} k_{1}^{\prime} \mathbf{t}+\left(k_{1}^{3}+k_{1}^{\prime \prime}+k_{1} k_{2}^{2}\right) \mathbf{n}+\left(2 k_{1}^{\prime} k_{2}+k_{1} k_{2}^{\prime}\right) \mathbf{b}_{1}+k_{1} k_{2} k_{3} \mathbf{b}_{2}$.

Using the above equations, the third curvature of $\alpha$ can be found by $k_{3}=-\frac{g\left(\alpha^{(4)}, \mathbf{b}_{2}\right)}{k_{1} k_{2}}$ for Case 1.1 and $k_{3}=\frac{g\left(\alpha^{(4)}, \mathbf{b}_{2}\right)}{k_{1} k_{2}}$ for Case 1.2 and Case 2.

3. The curvatures of spacelike intersection curve. Let $M_{i}(i=1,2,3)$ be three spacelike hypersurfaces with parametric equations $X^{i}=$ $X^{i}\left(u_{i}, v_{i}, w_{i}\right)$. Let us assume these hypersurfaces intersect transversally at an intersection point $\alpha\left(s_{0}\right)=P$ on the spacelike intersection curve $\alpha(s)$. 
Then, the unit normal vectors $\mathbf{N}_{i}$ of these hypersurfaces are timelike vectors and they can be given by

$$
\mathbf{N}_{i}=\frac{X_{u_{i}}^{i} \otimes X_{v_{i}}^{i} \otimes X_{w_{i}}^{i}}{\left\|X_{u_{i}}^{i} \otimes X_{v_{i}}^{i} \otimes X_{w_{i}}^{i}\right\|} .
$$

Since the intersection curve $\alpha$ is a spacelike curve, the unit tangent vector $\mathbf{t}$ of $\alpha$ is a spacelike vector and can be obtained by the ternary product of the normal vectors at $P$ :

$$
\mathbf{t}=\frac{\mathbf{N}_{1} \otimes \mathbf{N}_{2} \otimes \mathbf{N}_{3}}{\left\|\mathbf{N}_{1} \otimes \mathbf{N}_{2} \otimes \mathbf{N}_{3}\right\|}
$$

Since the intersection is transversal, the normal vectors $\mathbf{N}_{i}$ are linearly independent at the intersection point, i.e., $\mathbf{N}_{1} \otimes \mathbf{N}_{2} \otimes \mathbf{N}_{3} \neq 0$.

3.1. First Curvature. From the Frenet equations, we know $k_{1}=\left\|\mathbf{t}^{\prime}\right\|$. Then we must find the vector $\mathbf{t}^{\prime}$ for calculating the first curvature $k_{1}$. Since $\mathbf{t}^{\prime}$ is orthogonal to $\mathbf{t}$, we can write

$$
\alpha^{\prime \prime}=\mathbf{t}^{\prime}=a_{1} \mathbf{N}_{1}+a_{2} \mathbf{N}_{2}+a_{3} \mathbf{N}_{3}, a_{i} \in \mathbb{R} .
$$

Now let us determine the scalars $a_{i}$ to find $\alpha^{\prime \prime}$. If we take the dot product of both hand sides of (3.1) with $\mathbf{N}_{i}$, then we get

$$
g\left(\mathbf{N}_{1}, \mathbf{N}_{\mathbf{i}}\right) a_{1}+g\left(\mathbf{N}_{2}, \mathbf{N}_{\mathbf{i}}\right) a_{2}+g\left(\mathbf{N}_{3}, \mathbf{N}_{\mathbf{i}}\right) a_{3}=K_{n}^{i},
$$

where $K_{n}^{i}=g\left(\mathbf{t}^{\prime}, \mathbf{N}_{i}\right),(i=1,2,3)$. The coefficients determinant of the linear system (3.2) is $\Delta=-\left\|\mathbf{N}_{1} \otimes \mathbf{N}_{2} \otimes \mathbf{N}_{3}\right\|^{2}$. Since $\Delta$ is different from zero, solving the coefficients from linear system (3.2) yields (as in [4])

$$
a_{i}=\frac{1}{\Delta}\left\{-\sinh ^{2} \theta_{j k} K_{n}^{i}+b_{i j} K_{n}^{j}+b_{i k} K_{n}^{k}\right\}, \quad i, j, k=1,2,3 \text { (cyclic), }
$$

where $\theta_{i j}$ is the angle between the timelike unit normal vectors $\mathbf{N}_{i}$ and $\mathbf{N}_{j}$. Also, if $\mathbf{N}_{1}, \mathbf{N}_{2}$ and $\mathbf{N}_{3}$ are in the same timecone of $E_{1}^{4}$, then

$$
b_{i j}=\cosh \theta_{i k} \cosh \theta_{j k}-\cosh \theta_{i j} \text {. }
$$

If $\mathbf{N}_{1}, \mathbf{N}_{2}$ and $\mathbf{N}_{3}$ are not in the same timecone of $E_{1}^{4}$, then

$$
b_{i j}=\cosh \theta_{i k} \cosh \theta_{j k}+\cosh \theta_{i j} .
$$

If $\mathbf{N}_{1}$ and $\mathbf{N}_{2}$ are in the same timecone but $\mathbf{N}_{3}$ is in the different timecone, then

$$
b_{i j}=(-1)^{i+k} \cosh \theta_{i k} \cosh \theta_{j k}-\cosh \theta_{i j} .
$$

If $\mathbf{N}_{1}$ and $\mathbf{N}_{3}$ are in the same timecone but $\mathbf{N}_{2}$ is in the different timecone, then

$$
b_{i j}=(-1)^{i+j} \cosh \theta_{i k} \cosh \theta_{j k}-\cosh \theta_{i j} .
$$

If $\mathbf{N}_{2}$ and $\mathbf{N}_{3}$ are in the same timecone but $\mathbf{N}_{1}$ is in the different timecone, then

$$
b_{i j}=(-1)^{j+k} \cosh \theta_{i k} \cosh \theta_{j k}-\cosh \theta_{i j} .
$$


The normal curvatures $K_{n}^{i}$ needed to find the scalars $a_{i}$ are calculated as expressed in [4]. Then the first curvature $k_{1}$ of the intersection curve $\alpha$ at $P$ is given as follows:

If $\mathbf{N}_{1}, \mathbf{N}_{2}$ and $\mathbf{N}_{3}$ are in the same timecone, then

$$
k_{1}^{2}=\left|a_{1}^{2}+a_{2}^{2}+a_{3}^{2}+2 a_{1} a_{2} \cosh \theta_{12}+2 a_{1} a_{3} \cosh \theta_{13}+2 a_{2} a_{3} \cosh \theta_{23}\right| .
$$

If $\mathbf{N}_{1}, \mathbf{N}_{2}$ and $\mathbf{N}_{3}$ are not in the same timecone, then

$$
k_{1}^{2}=\left|a_{1}^{2}+a_{2}^{2}+a_{3}^{2}-2 a_{1} a_{2} \cosh \theta_{12}-2 a_{1} a_{3} \cosh \theta_{13}-2 a_{2} a_{3} \cosh \theta_{23}\right| .
$$

If $\mathbf{N}_{1}$ and $\mathbf{N}_{2}$ are in the same timecone but $\mathbf{N}_{3}$ is in the different timecone, then

$$
k_{1}^{2}=\left|a_{1}^{2}+a_{2}^{2}+a_{3}^{2}+2 a_{1} a_{2} \cosh \theta_{12}-2 a_{1} a_{3} \cosh \theta_{13}-2 a_{2} a_{3} \cosh \theta_{23}\right| .
$$

If $\mathbf{N}_{1}$ and $\mathbf{N}_{3}$ are in the same timecone but $\mathbf{N}_{2}$ is in the different timecone, then

$$
k_{1}^{2}=\left|a_{1}^{2}+a_{2}^{2}+a_{3}^{2}-2 a_{1} a_{2} \cosh \theta_{12}+2 a_{1} a_{3} \cosh \theta_{13}-2 a_{2} a_{3} \cosh \theta_{23}\right| .
$$

If $\mathbf{N}_{2}$ and $\mathbf{N}_{3}$ are in the same timecone but $\mathbf{N}_{1}$ is in the different timecone, then

$$
k_{1}^{2}=\left|a_{1}^{2}+a_{2}^{2}+a_{3}^{2}-2 a_{1} a_{2} \cosh \theta_{12}-2 a_{1} a_{3} \cosh \theta_{13}+2 a_{2} a_{3} \cosh \theta_{23}\right| .
$$

3.2. Second Curvature. Now, let us find the second curvature of the intersection curve $\alpha$ at $P$. Since the timelike unit normal vectors $\mathbf{N}_{i}$ are orthogonal to $\mathbf{t}$, the terms $k_{1}^{\prime} \mathbf{n}+k_{1} k_{2} \mathbf{b}_{1}$ in Eq. (2.1) and Eq. (2.2) can be replaced by $c_{1} \mathbf{N}_{1}+c_{2} \mathbf{N}_{2}+c_{3} \mathbf{N}_{3}$. Thus

Case 1: $\alpha^{\prime \prime \prime}=-k_{1}^{2} \mathbf{t}+c_{1} \mathbf{N}_{1}+c_{2} \mathbf{N}_{2}+c_{3} \mathbf{N}_{3}$,

Case 2: $\alpha^{\prime \prime \prime}=k_{1}^{2} \mathbf{t}+c_{1} \mathbf{N}_{1}+c_{2} \mathbf{N}_{2}+c_{3} \mathbf{N}_{3}$.

If the dot products of both hand sides of above equations are taken with $\mathbf{N}_{i}$, we have a linear equation system similar to (3.2). Solving this system yields

$$
c_{i}=\frac{1}{\Delta}\left\{-\sinh ^{2} \theta_{j k} \mu_{i}+b_{i j} \mu_{j}+b_{i k} \mu_{k}\right\}, \quad i, j, k=1,2,3 \text { (cyclic), }
$$

where $\mu_{i}=g\left(\alpha^{\prime \prime \prime}, \mathbf{N}_{i}\right)$. The scalars $\mu_{i}$ in Eq. (3.3) are computed as explained in [4]. Then, the second curvature $k_{2}$ of the intersection curve $\alpha$ at $P$ is given by

Case 1.1 and Case $2: k_{2}=\frac{g\left(\alpha^{\prime \prime \prime}, \mathbf{b}_{1}\right)}{k_{1}}$,

Case 1.2: $k_{2}=-\frac{g\left(\alpha^{\prime \prime \prime}, \mathbf{b}_{1}\right)}{k_{1}}$.

3.3. Third Curvature. Now, let us compute the third curvature $k_{3}$ of the curve $\alpha$ at $P$. Similar to the second and third derivatives of $\alpha$, we may write

Case 1: $\alpha^{(4)}=-3 k_{1} k_{1}^{\prime} \mathbf{t}+d_{1} \mathbf{N}_{1}+d_{2} \mathbf{N}_{2}+d_{3} \mathbf{N}_{3}$, 
Case 2: $\alpha^{(4)}=3 k_{1} k_{1}^{\prime} \mathbf{t}+d_{1} \mathbf{N}_{1}+d_{2} \mathbf{N}_{2}+d_{3} \mathbf{N}_{3}$, where

$$
d_{i}=\frac{1}{\Delta}\left\{-\sinh ^{2} \theta_{j k} \xi_{i}+b_{i j} \xi_{j}+b_{i k} \xi_{k}\right\}, \quad i, j, k=1,2,3 \text { (cyclic), }
$$

and $\xi_{i}=g\left(\alpha^{(4)}, \mathbf{N}_{i}\right)$. Obtaining the scalars $\xi_{i}$ is mentioned in [4].

Thus, the third curvature of the intersection curve is found as

Case 1.1: $k_{3}=-\frac{g\left(\alpha^{(4)}, \mathbf{b}_{2}\right)}{k_{1} k_{2}}$,

Case 1.2 and Case 2: $k_{3}=\frac{g\left(\alpha^{(4)}, \mathbf{b}_{2}\right)}{k_{1} k_{2}}$.

\section{Examples.}

4.1. Example 1. Let us consider the spacelike hypersurfaces

$$
\begin{aligned}
M_{1}: X^{1}\left(u_{1}, v_{1}, w_{1}\right)=\left(\cosh u_{1}+\frac{1}{2}, \sinh u_{1}, v_{1}, w_{1}\right), \\
M_{2}: X^{2}\left(u_{2}, v_{2}, w_{2}\right)=\left(\cosh u_{2}, \sinh u_{2} \cos v_{2}, \sinh u_{2} \sin v_{2}, w_{2}\right), \\
M_{3}: X^{3}\left(u_{3}, v_{3}, w_{3}\right) \\
\quad=\left(\frac{1}{\sqrt{2}} \cosh u_{3}+\frac{1}{2}, \frac{1}{\sqrt{2}} \sinh u_{3} \sin v_{3}+\frac{1}{2}, w_{3}, \frac{1}{\sqrt{2}} \sinh u_{3} \cos v_{3}\right)
\end{aligned}
$$

in the Minkowski 4 -space, where $u_{2} \neq 0, u_{3} \neq 0$. Let us compute the Frenet apparatus of the spacelike intersection curve $\alpha$ at the intersection point

$$
\begin{aligned}
P & =X^{1}\left(0, \frac{\sqrt{5}}{2}, \frac{1}{2}\right)=X^{2}\left(\ln \left(\frac{3+\sqrt{5}}{2}\right), \frac{\pi}{2}, \frac{1}{2}\right) \\
& =X^{3}\left(\ln (\sqrt{2}+1), \frac{7 \pi}{4}, \frac{\sqrt{5}}{2}\right)=\left(\frac{3}{2}, 0, \frac{\sqrt{5}}{2}, \frac{1}{2}\right) .
\end{aligned}
$$

At this point, the timelike unit normal vectors of these hypersurfaces are, respectively,

$$
\mathbf{N}_{1}=(1,0,0,0), \mathbf{N}_{2}=\left(\frac{3}{2}, 0, \frac{\sqrt{5}}{2}, 0\right), \mathbf{N}_{3}=\left(\sqrt{2},-\frac{1}{\sqrt{2}}, 0, \frac{1}{\sqrt{2}}\right)
$$

and the spacelike unit tangent vector of $\alpha$ is

$$
\mathbf{t}=\left(0, \frac{1}{\sqrt{2}}, 0, \frac{1}{\sqrt{2}}\right) .
$$

Since $g\left(\mathbf{N}_{1}, \mathbf{N}_{2}\right), g\left(\mathbf{N}_{1}, \mathbf{N}_{3}\right)$ and $g\left(\mathbf{N}_{2}, \mathbf{N}_{3}\right)$ are smaller than zero, the timelike unit normal vectors $\mathbf{N}_{i}, 1 \leq i \leq 3$, are in the same timecone. Then, $\cosh \theta_{12}=\frac{3}{2}, \cosh \theta_{13}=\sqrt{2}, \cosh \theta_{23}=\frac{3}{\sqrt{2}}$ and so $b_{12}=\frac{3}{2}, b_{13}=\frac{5}{2 \sqrt{2}}$ and $b_{23}=0$. 
The non-vanishing first fundamental form coefficients of these hypersurfaces at $P$ are

$$
g_{11}^{1}=g_{22}^{1}=g_{33}^{1}=g_{11}^{2}=g_{33}^{2}=g_{33}^{3}=1, g_{22}^{2}=\frac{5}{4}, g_{11}^{3}=g_{22}^{3}=\frac{1}{2}
$$

and the non-vanishing second fundamental form coefficients of these hypersurfaces at $P$ are

$$
h_{11}^{1}=h_{11}^{2}=-1, h_{22}^{2}=-\frac{5}{4}, h_{11}^{3}=h_{22}^{3}=-\frac{1}{\sqrt{2}} .
$$

As expressed in [4], the values $u_{i}^{\prime}, v_{i}^{\prime}$ and $w_{i}^{\prime}$ are found as:

$$
\begin{array}{rlrl}
u_{1}^{\prime} & =\frac{1}{\sqrt{2}}, & v_{1}^{\prime}=0, & w_{1}^{\prime}=\frac{1}{\sqrt{2}}, \\
u_{2}^{\prime}=0, & v_{2}^{\prime}=-\frac{\sqrt{2}}{\sqrt{5}}, & w_{2}^{\prime}=\frac{1}{\sqrt{2}}, \\
u_{3}^{\prime}=0, & v_{3}^{\prime}=\sqrt{2}, & w_{3}^{\prime}=0 .
\end{array}
$$

Hence, for the normal curvatures $K_{n}^{i}$ we find $K_{n}^{1}=K_{n}^{2}=-\frac{1}{2}, K_{n}^{3}=-\sqrt{2}$. Thus, we have

$$
\begin{aligned}
& a_{1}=\frac{6}{5}, \quad a_{2}=\frac{1}{5}, \quad a_{3}=-\frac{1}{\sqrt{2}}, \quad k_{1}=\frac{\sqrt{3}}{\sqrt{10}}, \\
& \alpha^{\prime \prime}=\left(\frac{1}{2}, \frac{1}{2}, \frac{1}{2 \sqrt{5}},-\frac{1}{2}\right), \quad \mathbf{n}=\left(\frac{\sqrt{5}}{\sqrt{6}}, \frac{\sqrt{5}}{\sqrt{6}}, \frac{1}{\sqrt{6}},-\frac{\sqrt{5}}{\sqrt{6}}\right),
\end{aligned}
$$

where $\mathbf{n}$ is a spacelike vector. Also, the values $u_{i}^{\prime \prime}, v_{i}^{\prime \prime}$ and $w_{i}^{\prime \prime}$ are calculated as:

$$
\begin{aligned}
u_{1}^{\prime \prime}=\frac{1}{2}, & v_{1}^{\prime \prime}=\frac{1}{2 \sqrt{5}}, & w_{1}^{\prime \prime}=-\frac{1}{2}, \\
u_{2}^{\prime \prime}=\frac{1}{\sqrt{5}}, & v_{2}^{\prime \prime}=-\frac{1}{\sqrt{5}}, & w_{2}^{\prime \prime}=-\frac{1}{2}, \\
u_{3}^{\prime \prime}=\frac{1}{\sqrt{2}}, & v_{3}^{\prime \prime}=0, & w_{3}^{\prime \prime}=\frac{1}{2 \sqrt{5}} .
\end{aligned}
$$

Using above, we get $\mu_{1}=\mu_{2}=-\frac{3}{2 \sqrt{2}}$ and $\mu_{3}=0$. So, the coefficients $c_{i}$ are found as $c_{1}=-\frac{12}{5 \sqrt{2}}, c_{2}=\frac{3}{5 \sqrt{2}}, c_{3}=\frac{3}{2}$. Then, we get

$$
\begin{aligned}
& \alpha^{\prime \prime \prime}=\left(\frac{3}{2 \sqrt{2}},-\frac{9}{5 \sqrt{2}}, \frac{3 \sqrt{5}}{10 \sqrt{2}}, \frac{6}{5 \sqrt{2}}\right), \\
& \alpha^{\prime} \otimes \alpha^{\prime \prime} \otimes \alpha^{\prime \prime \prime}=\left(\frac{3}{2 \sqrt{5}}, 0, \frac{3}{2}, 0\right), \quad\left\|\alpha^{\prime} \otimes \alpha^{\prime \prime} \otimes \alpha^{\prime \prime \prime}\right\|=\frac{3}{\sqrt{5}},
\end{aligned}
$$




$$
\begin{aligned}
& \mathbf{b}_{2}=\frac{\alpha^{\prime} \otimes \alpha^{\prime \prime} \otimes \alpha^{\prime \prime \prime}}{\left\|\alpha^{\prime} \otimes \alpha^{\prime \prime} \otimes \alpha^{\prime \prime \prime}\right\|}=\left(\frac{1}{2}, 0, \frac{\sqrt{5}}{2}, 0\right), \\
& \mathbf{b}_{1}=\frac{\mathbf{b}_{2} \otimes \alpha^{\prime} \otimes \alpha^{\prime \prime}}{\left\|\mathbf{b}_{2} \otimes \alpha^{\prime} \otimes \alpha^{\prime \prime}\right\|}=\left(\frac{5}{2 \sqrt{3}}, \frac{1}{\sqrt{3}}, \frac{\sqrt{5}}{2 \sqrt{3}},-\frac{1}{\sqrt{3}}\right) .
\end{aligned}
$$

Note that $\mathbf{b}_{2}$ is a spacelike vector and $\mathbf{b}_{1}$ is the unique timelike vector in the tetrad $\left\{\mathbf{t}, \mathbf{n}, \mathbf{b}_{1}, \mathbf{b}_{2}\right\}$. Because of this, Case 1.2 is valid. Then the second curvature $k_{2}$ of $\alpha$ is found as $k_{2}=2 \sqrt{5}$. Also, $k_{1}^{\prime}=g\left(\alpha^{\prime \prime \prime}, \mathbf{n}\right)=-\frac{7 \sqrt{15}}{10}$.

For the values $u_{i}^{\prime \prime \prime}, v_{i}^{\prime \prime \prime}$ and $w_{i}^{\prime \prime \prime}$, we obtain

$$
\begin{array}{lll}
u_{1}^{\prime \prime \prime}=-\frac{23}{10 \sqrt{2}}, & v_{1}^{\prime \prime \prime}=\frac{3 \sqrt{5}}{10 \sqrt{2}}, & w_{1}^{\prime \prime \prime}=\frac{6}{5 \sqrt{2}}, \\
u_{2}^{\prime \prime \prime}=\frac{3}{\sqrt{10}}, & v_{2}^{\prime \prime \prime}=\frac{32}{5 \sqrt{10}}, & w_{2}^{\prime \prime \prime}=\frac{6}{5 \sqrt{2}}, \\
u_{3}^{\prime \prime \prime}=\frac{3}{2}, & v_{3}^{\prime \prime \prime}=-\frac{13}{5 \sqrt{2}}, & w_{3}^{\prime \prime \prime}=\frac{3}{2 \sqrt{10}} .
\end{array}
$$

Hence, we have

$$
\xi_{1}=\frac{18}{5}, \quad \xi_{2}=\frac{69}{20}, \quad \xi_{3}=-\frac{21}{10 \sqrt{2}}
$$

and using these values

$$
d_{1}=\frac{201}{25}, \quad d_{2}=-\frac{39}{25}, \quad d_{3}=-\frac{93}{10 \sqrt{2}}
$$

are found. So, we obtain

$$
\begin{aligned}
\alpha^{(4)} & =\left(-\frac{18}{5}, \frac{38}{5},-\frac{39}{10 \sqrt{5}},-\frac{3}{2}\right), \\
k_{3} & =-\frac{3}{20 \sqrt{6}} .
\end{aligned}
$$

4.2. Example 2. Let $M_{1}, M_{2}$, and $M_{3}$ be the spacelike hypersurfaces given by, respectively,

$$
\begin{aligned}
& X^{1}\left(u_{1}, v_{1}, w_{1}\right)=\left(\cosh u_{1}, \sinh u_{1}+\frac{1}{2}, v_{1}, w_{1}\right), \\
& X^{2}\left(u_{2}, v_{2}, w_{2}\right)=\left(\frac{1}{2} \cosh u_{2}, \frac{1}{2} \sinh u_{2} \cos v_{2}, w_{2}, \frac{1}{2} \sinh u_{2} \sin v_{2}\right), \\
& X^{3}\left(u_{3}, v_{3}, w_{3}\right)=\left(\frac{1}{2} \cosh u_{3}, w_{3}, \frac{1}{2} \sinh u_{3} \sin v_{3}-\frac{1}{2}, \frac{1}{2} \sinh u_{3} \cos v_{3}\right),
\end{aligned}
$$


where $u_{2} \neq 0, u_{3} \neq 0$. The Frenet vectors and the curvatures of the spacelike intersection curve of these surfaces at the intersection point

$$
\begin{aligned}
P & =X^{1}\left(0,0, \frac{1}{\sqrt{2}}\right)=X^{2}(\ln (2+\sqrt{3}), \arctan \sqrt{2}, 0) \\
& =X^{3}\left(\ln (2+\sqrt{3}), \arctan \frac{1}{\sqrt{2}}, \frac{1}{2}\right)=\left(1, \frac{1}{2}, 0, \frac{1}{\sqrt{2}}\right)
\end{aligned}
$$

are found as:

$$
\begin{aligned}
& \mathbf{t}=\left(0,-\frac{\sqrt{2}}{\sqrt{5}},-\frac{\sqrt{2}}{\sqrt{5}}, \frac{1}{\sqrt{5}}\right), \quad \mathbf{n}=\left(\frac{5}{\sqrt{15}},-\frac{1}{\sqrt{15}},-\frac{1}{\sqrt{15}},-\frac{2 \sqrt{2}}{\sqrt{15}}\right), \\
& \mathbf{b}_{1}=\left(-\frac{\sqrt{2}}{\sqrt{3}}, \frac{1}{\sqrt{6}}, \frac{1}{\sqrt{6}}, \frac{2}{\sqrt{3}}\right), \quad \mathbf{b}_{2}=\left(0,-\frac{1}{\sqrt{2}}, \frac{1}{\sqrt{2}}, 0\right), \\
& k_{1}=\frac{2 \sqrt{15}}{25}, \quad k_{2}=2, \quad k_{3}=0 .
\end{aligned}
$$

Here, the timelike unit normal vectors $\mathbf{N}_{2}$ and $\mathbf{N}_{3}$ are in the same timecone but $\mathbf{N}_{1}$ is in the different timecone. Also, $\mathbf{n}$ is the unique timelike vector in the Frenet frame $\left\{\mathbf{t}, \mathbf{n}, \mathbf{b}_{1}, \mathbf{b}_{2}\right\}$ and for this reason, Case 2 is valid.

\section{REFERENCES}

[1] Alessio, O., Geometria diferencial de curvas de interseção de duas superfícies implícitas, TEMA Tend. Mat. Apl. Comput. 7 (2) (2006), 169-178.

[2] Alessio, O., Guadalupe, I. V., Determination of a transversal intersection curve of two spacelike surfaces in Lorentz-Minkowski 3-Space $L^{3}$, Hadronic Journal 30 (3) (2007), 315-342.

[3] Alessio, O., Differential geometry of intersection curves in $R^{4}$ of three implicit surfaces, Comput. Aided Geom. Des. 26 (2009), 455-471.

[4] Düldül, M., On the intersection curve of three parametric hypersurfaces, Comput. Aided Geom. Des. 27 (2010), 118-127.

[5] Goldman, R., Curvature formulas for implicit curves and surfaces, Comput. Aided Geom. Des. 22 (2005), 632-658.

[6] Hartmann, E., $G^{2}$ interpolation and blending on surfaces, The Visual Computer 12 (1996), 181-192.

[7] Turgut, A., Spacelike and timelike ruled surfaces on the Minkowski 3-space $R_{1}^{3}, \mathrm{Ph}$. D. thesis, Ankara University, 1995.

[8] O'Neill, B., Semi Riemannian Geometry, Academic Press, New York-London, 1983.

[9] Walrave, J., Curves and surfaces in Minkowski space, Ph. D. thesis, K. U. Leuven. Fac. Science, Leuven, 1995.

[10] Williams, M. Z., Stein, F. M., A triple product of vectors in four-space, Math. Mag. 37 (4) (1964), 230-235.

[11] Willmore, T. J., An Introduction to Differential Geometry, Clarendon Press, Oxford, 1959.

[12] Ye, X., Maekawa T., Differential geometry of intersection curves of two surfaces, Comput. Aided Geom. Des. 16 (1999), 767-788.

[13] Yilmaz, S., Turgut, M., On the differential geometry of the curves in Minkowski space-time I, Int. J. Contemp. Math. Sciences 3 (27) (2008), 1343-1349. 
B. Uyar Düldül

Department of Mathematics Education

Education Faculty

Yıldız Technical University

İstanbul

Turkey

e-mail: buduldul@yildiz.edu.tr

Received February 2, 2012
M. Çalışkan

Department of Mathematics

Science Faculty

Gazi University

Ankara

Turkey

e-mail: mustafacaliskan@gazi.edu.tr 